\title{
AVALIAÇÃO DO CONHECIMENTO SOBRE PLANTAS ALIMENTÍCIAS NÃO CONVENCIONAIS (PANC) POR MEIO DE QUESTIONÁRIO DA PLATAFORMA GOOGLE FORMS APLICADO À POPULAÇÃO
}

\author{
Luiza Varela Nunes ${ }^{1}$ \\ Juliana Audi Giannoni2 ${ }^{2}$ \\ Alef Goes Costa ${ }^{3}$ \\ Alaide Carvalho Ferreira ${ }^{4}$ \\ Pedro Henrique Silva de Rossi ${ }^{5}$ \\ Silvana Pedroso de Góes Favoni'
}

RESUMO: Um quadro como o qual o Brasil se encontra atualmente, com volume de produção elevadíssimo de uma variedade muito pequena de alimentos, entra em contradição com os dados de fome e desnutrição observados ao longo de todo o país, e principalmente evidenciados em regiões mais carentes. Isso acontece porque, muitas vezes, as monoculturas não são capazes de garantir a segurança alimentar à toda população. A segurança alimentar é alcançada quando todas as pessoas, em todos os momentos, tenham acesso físico e econômico a alimentos que garantam sua exigência nutricional, permitindo uma vida saudável (ABREU; CASTANHEIRA, 2017).

\footnotetext{
'Engenheira Biotecnológica pela Universidade Estadual Paulista "Júlio de Mesquita Filho"; Graduanda em Tecnologia em Alimentos pela Faculdade de Tecnologia de Marília (FATEC). E-mail: luizavarela.n@gmail.com

2 Pós-Doutorado em Ciência dos Alimentos com Irradiação Gama e Processamento Mínimo pela Universidade Federal de Lavras (UFLA), Lavras/MG. Doutorado e Mestrado em Agronomia/Horticultura pela Universidade Estadual Paulista "Júlio de Mesquita Filho" Campus Botucatu-SP. Graduação em Agronomia pela Universidade Federal de Lavras (UFLA), Lavras/MG. Professora Associado I, junto ao curso de Tecnologia em Alimentos e da Pós-Graduação da FATEC/Marília/SP. Experiência na área de Ciência e Tecnologia de Alimentos, atuando principalmente nos seguintes temas: Toxicologia, Processamento mínimo, Irradiação Gama, Análise sensorial, Tecnologia de Açúcar e Álcool; e na área de Horticultura com PANCs.E-mail: juliana.giannonior@fatec.sp.gov.br

${ }^{3}$ Graduação em Tecnologia em Alimentos pela Faculdade de Tecnologia de Marília (FATEC). E-mail: alefgoes2@gmail.com

${ }^{4}$ Graduação em Tecnologia em Alimentos pela Faculdade de Tecnologia de Marília (FATEC) E-mail: cfalaide@gmail.com

${ }^{5}$ Tecnólogo em Alimentos pela Faculdade de Tecnologia de Marília (FATEC). Discente e Pesquisador do curso de Tecnologia em Alimentos da Faculdade de Tecnologia de Marília. Revisor de periódicos internacionais. Possui certificado da Academia Publons sobre graduação de revisores por pares. Tem experiência na área de Saúde Coletiva. E-mail:pps.20ro.ph@gmail.com

${ }^{6}$ Doutorado em Ciência de Alimentos pela Universidade Estadual de Londrina (UEL), Especialização em Bioquímica Aplicada (UEL), Graduação em Ciências Biológicas (UEL). Atualmente é professora titular do Centro Estadual de Educação Tecnológica Paula Souza - FATEC Marilia. Tem experiência na área de Bioquímica, Biotecnologia, Controle de Qualidade. E-mail: silvana.favoni2@fatec.sp.gov.br
} 
Palavra-chave: Alimento. Plantas Alimentícias. Plantas.

\section{INTRODUÇÃO}

A maior parte das calorias consumidas na dieta da população brasileira se baseia em alguns poucos vegetais que formam os pilares da monocultura no país, como arroz, soja, milho, trigo, cana-de-açúcar, feijão, batata, mandioca, banana e amendoim. Por consequência da prática da monocultura e da priorização da indústria agrícola por alguns cultivares de maior produtividade e rentabilidade, muitas caraterísticas também importantes nos alimentos como nutrientes, funcionalidade, cor, sabor e forma acabam sendo deixadas de lado em programas de melhoramento e incentivo e a dieta da população acaba se tornando cada vez mais monótona (BEZERRA; BRITO, 2020).

A globalização do mercado alimentar levou a um afunilamento de commodities produzidas no Brasil, tido como um dos líderes na produção global de alimentos, e que acaba refletindo nos produtos que são consumidos nacional e regionalmente, uma vez que tomam espaço da produção de subsistência. Tal processo de simplificação da dieta alimentar da população e o abandono da produção de subsistência leva a um processo denominado erosão cultural alimentar. Com isso, a produção nacional sofre uma perda significativa e gradativa de uma alimentação variada, complexa e vinculada à cultura local e ao ambiente. O processo de erosão cultural alimentar induz à subutilização de espécies locais que evoluíram conjuntamente ao homem e, por consequência, diversas plantas que poderiam ser cultivadas acabam sendo descartadas e tratadas como ervas daninhas (FONSECA et al., 2018).

Um quadro como o qual o Brasil se encontra atualmente, com volume de produção elevadíssimo de uma variedade muito pequena de alimentos, entra em contradição com os dados de fome e desnutrição observados ao longo de todo o país, e principalmente evidenciados em regiões mais carentes. Isso acontece porque, muitas vezes, as monoculturas não são capazes de garantir a segurança alimentar à toda população. A segurança alimentar é alcançada quando todas as pessoas, em todos os momentos, tenham acesso físico e econômico a alimentos que garantam sua exigência nutricional, permitindo uma vida saudável (ABREU; CASTANHEIRA, 2017). 
Muitas das chamadas ervas daninhas e das espécies que são descartadas no momento de plantio e colheita são na realidade plantas alimentícias não convencionais (PANCs). As PANCs são aquelas espécies comestíveis que não estão inclusas na cadeia produtiva e que possuem distribuição limitada a certas localidades, capazes de influenciar a cultura alimentar da região (KINUPP, 2009). Também passam a ser classificadas como PANCs aquelas plantas cujo consumo está restrito a apenas uma parte do vegetal, sendo as demais partes comestíveis descartadas (REIS et al., 2016).

Apesar de sua distribuição restrita e do pouco conhecimento da população como um todo sobre seus benefícios, as PANCs têm grande potencial nutricional e funcional, além de contribuírem para o fortalecimento da biodiversidade. Muitas espécies se mostram ricas em fibras, vitaminas e sais minerais, se encaixando como boas opções na dieta humana. Tais espécies apresentam muitas possibilidades de uso na alimentação, algumas sendo consumidas in natura, minimamente processadas ou como constituintes de processamento e ultra processamento, contribuindo para sua inclusão na dieta (JACOB, 2020).

Essas espécies destacam-se, ainda, por uma composição muito rica de compostos fitoquímicos funcionais, que são os casos de flavonoides e carotenoides. Tais substâncias são entendidas mundialmente como capazes de apresentar propriedades anti-inflamatórias, antimicrobianas, antioxidantes e anticarcinogênicas, auxiliando na prevenção de doenças crônicas e possibilitando uma melhor qualidade de vida. Os alimentos funcionais são aqueles que proporcionam efeitos metabólicos e fisiológicos benéficos à saúde, e diversas PANCs podem se encaixar nessa descrição, desde que comprovada a segurança do seu consumo e suas propriedades (FONSECA et al., 2018; SILVA et al., 2019).

Apesar dos inúmeros benefícios à saúde, à biodiversidade, à sociedade e à cultura alimentar regional, as PANCs ainda não têm espaço significativo no cotidiano da grande maioria da população brasileira, devido, principalmente, à falta de conhecimento. Mostrase extremamente necessária a realização de pesquisas e estudos que promovam a expansão do conhecimento popular sobre as espécies locais e suas propriedades e usos, contribuindo, assim, para a soberania alimentar. 
O presente trabalho teve como objetivo avaliar o conhecimento popular sobre plantas alimentícias não convencionais (PANCs) através de uma pesquisa on-line realizada no Google Forms, bem como servir de material informativo e didático sobre o assunto, buscando esclarecer para a população atingida os benefícios e possibilidades das PANCs na dieta.

\section{METODOLOGIAS}

Foi aplicada uma pesquisa com abordagens de aspecto quantitativo e qualitativo referentes a plantas alimentícias não convencionais ou PANCs. O questionário foi elaborado na plataforma Google Forms e ficou disponível para o recebimento de respostas durante o período de io de março a 20 de abril de 2021. A pesquisa foi enviada de maneira aleatória aos participantes através de meios de comunicação on-line. A mesma foi aplicada a 148 pessoas de todas as regiões do Brasil, de todas as idades e todos os níveis de escolaridade.

Além de perguntas para identificação do perfil dos participantes, o questionário apresentou uma lista com onze PANCs de diversas regiões do país, dentre elas Beldroega (Portulaca oleracea), Caruru (Amaranthus viridis), Fava (Vicia faba), Feijão guandu ou andu (Cajanus cajan), Folha de batata-doce (Ipomoea batatas), Ora-pro-nóbis (Pereskia aculeata), Palma (Opuntia ficus-indica), Peixinho-da-horta (Stachys byzantina), Picão (Bidens alba), Taioba (Xanthosoma sagittifolium) e Hibisco (Hibiscus sp). Além do nome popular e nome científico de cada planta, os participantes tiveram acesso a uma foto de cada uma das PANCs para melhor identificação.

Aqueles que aceitaram participar da pesquisa foram informados sobre os objetivos acadêmicos do estudo. As questões foram formuladas baseando-se em artigos científicos consultados nas bases de dados Google Acadêmico, PubMed e Lilacs e pelos próprios integrantes da pesquisa.

\section{RESULTADOS E DISCUSSÃO}


Os participantes foram questionados sobre o conhecimento do termo plantas alimentícias não convencionais, ou PANCs. 71,6\% dos entrevistados alegaram não terem conhecimento sobre nenhum dos termos. Tal valor é superior ao encontrado no estudo de Narcisa-Oliveira et al (2018) de 55\% de pessoas que desconheciam os termos. Uma parcela tão significativa de entrevistados que desconhecem a terminologia pode indicar a falta de informação, divulgação e incentivos para a inserção de PANCs na dieta. Ferrarini et al. (2020) encontrou um valor aproximado de 50\% para pessoas que não tinham conhecimento prévio sobre PANCs. Um valor mais alto encontrado no presente trabalho para pessoas que desconhecem o termo PANCs, quando comparado com os estudos de Narcisa-Oliveira et al (2018) e Ferrarini et al. (2020), pode ser causado pelo perfil de entrevistados, sendo que no presente trabalho a maior parte dos entrevistados alegaram ter entre 20 e 39 anos $(64,9 \%)$, serem do sexo feminino $(68,9 \%)$, nascidos nas regiões Sudeste e Sul $(77,7 \%)$ e possuírem ensino superior completo (52\%).

Observou-se que 29\% das pessoas entre 20 e 39 anos conhecem o termo PANC. Já entre os participantes de 40 a 59 anos, $34 \%$ conhecem o termo PANC. Entre os participantes acima de 60 anos, 9\% conhecem o termo PANC, e até 19 anos nenhum participante alegou conhecer o termo. Conforme Barreira et al. (2015) e Nedopetalski e Krupek (2020), o conhecimento sobre as PANCs se concentra mais em pessoas de mais idade, principalmente acima de 60 anos, que carregam a cultura regional e tradições familiares que são repassadas pelas gerações e que, em muitos casos, tem um maior contato com o campo. Contudo, as terminologias de plantas alimentícias não convencionais ou PANCs não necessariamente são conhecidas por pessoas dessa faixa etária. Seria interessante uma pesquisa mais aprofundada levando em consideração uma amostra maior de pessoas de cada faixa etária, para que se possa evidenciar com bases mais sólidas tais suposições.

Apesar da maioria das pessoas não conhecerem o termo PANCs, 59,4\% dos participantes alegaram ter consumido ao menos uma das PANCs listadas no estudo. 
Dentre as PANCs citadas na pesquisa, as mais conhecidas pelos entrevistados foram o hibisco, folha de batata-doce, picão, palma e feijão guandu, conforme ilustrado na figura I.

Figura 1. Total de pessoas que conhecem as PANCs citadas na pesquisa.

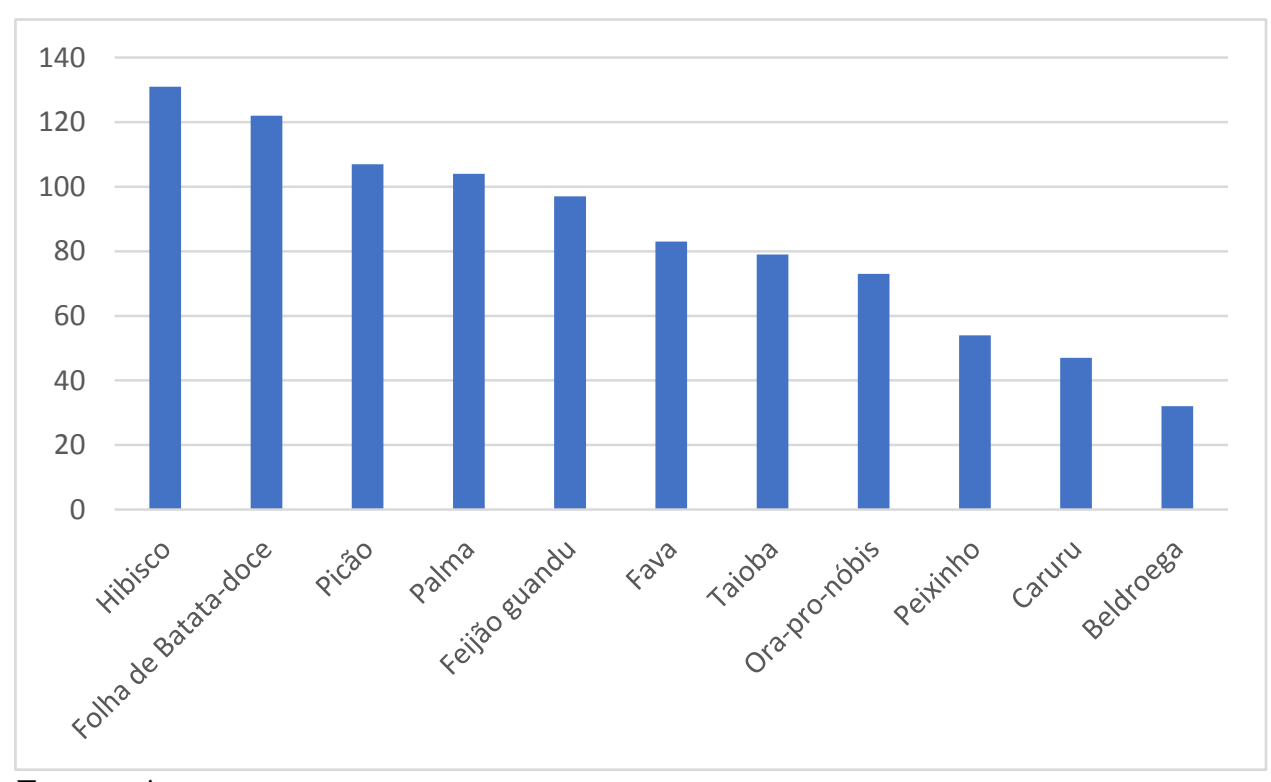

\section{Fonte: Autores}

Dentre as PANCs citadas na pesquisa, as mais consumidas pelos entrevistados são hibisco, feijão guandu, ora-pro-nóbis e picão, como visto na figura 2.

Figura 2. Total de pessoas que consomem as PANCs citadas na pesquisa.

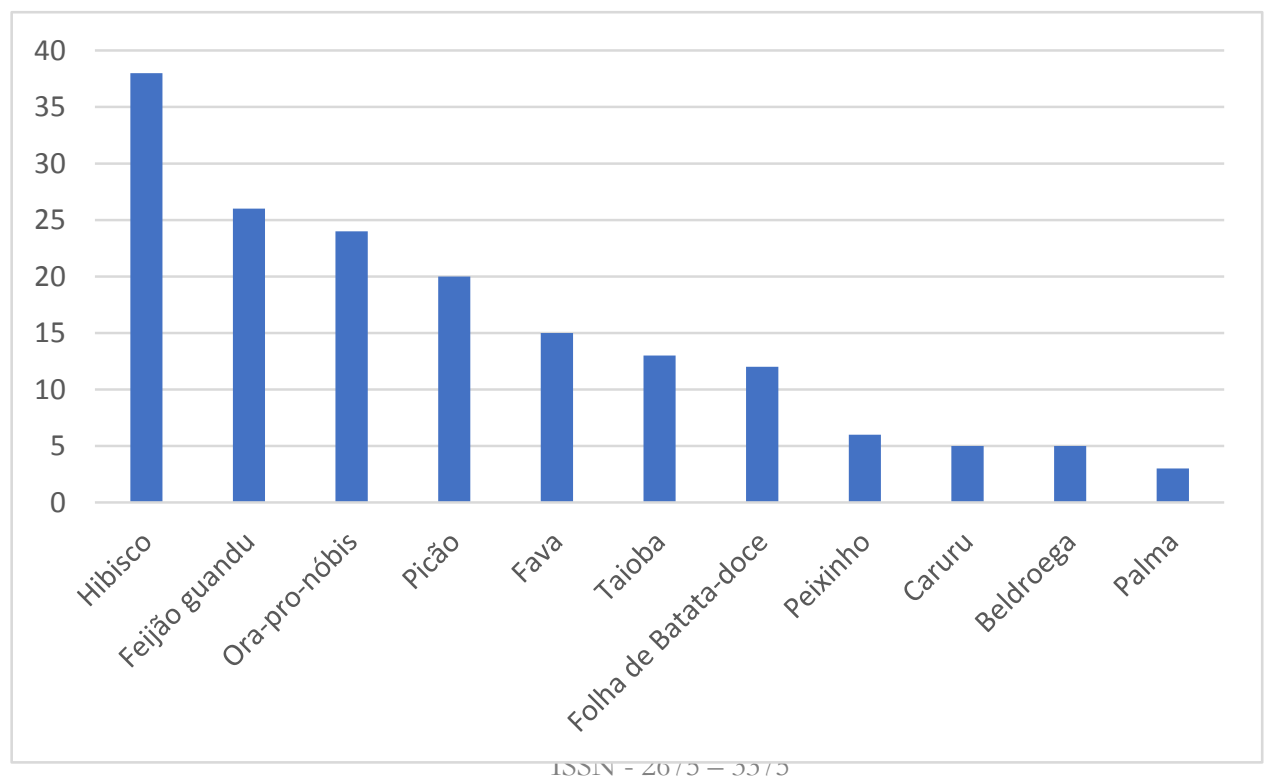




\section{Fonte: Autores}

Podemos perceber que as PANCs que são conhecidas pela população não são consumidas em igual proporção, com destaque especial para a palma, que é conhecida por $70,3 \%$ dos entrevistados, mas só é consumida por $2 \%$ deles.

O hibisco foi a PANC mais conhecida ( $88,5 \%$ dos entrevistados) e também a mais consumida (25,7\% dos entrevistados). A maioria das pessoas que alegaram ter consumido hibisco, fizeram esse consumo através de chás. O chá de hibisco, feito com as flores secas, é comumente utilizado em dietas com o objetivo de emagrecimento, sendo utilizado principalmente por mulheres. Alguns estudos já relacionaram o consumo de chá de hibisco com a redução do Índice de Massa Corporal (IMC) e de níveis de lipídios totais em indivíduos obesos. Tais efeitos estão relacionados à presença de antioxidantes, como a antocianina, e a um possível efeito termogênico (PRIETO et al., 2013). Suas flores também são usadas por muitos povos para o tratamento de problemas renais, pulmonares, gastrointestinais e infecções microbianas. Contudo, as demais partes da planta, como folhas, caules, raízes e sementes, também podem ser utilizadas na alimentação no preparo de cozidos, farinhas, sopas, sucos, geleias, molhos, sorvetes e licores (ALMEIDA; SCHWEIG, 2018; BARBOSA et al., 2021).

A ora-pro-nóbis é uma planta de pouca demanda hídrica e de baixa incidência de doenças, o que favorece seu cultivo doméstico. É um vegetal rico em proteínas, vitaminas, fibras e carotenoides, e suas folhas, flores e frutos são consumidos majoritariamente cozidos (BEZERRA; BRITO, 2020; GONÇALVES; LIMA; MORAES, 2020). Dentre as PANCs listadas na presente pesquisa, a ora-pro-nóbis se mostrou, proporcionalmente, ser aquela mais consumida quando comparado o número de consumidores e de pessoas que conheciam a planta $(32,9 \%$ das pessoas que conhecem a planta já fizeram seu consumo), seguida do hibisco ( $29 \%$ das pessoas que conhecem já fizeram o consumo). Tais dados estão em concordância com o estudo de Barbosa et al. (2021), que evidenciou que as pessoas participantes da referida pesquisa tinham disposição para consumir determinadas PANCs, sendo que dentre as plantas citadas as que geraram maior interesse foram a ora-pro-nóbis, 
a taioba e o hibisco. Ainda, o estudo indica que os motivos maiores para o consumo de tais plantas são a indicação da sabedoria popular ou familiar e o fácil acesso na região.

A palma apresenta uma considerável quantidade de fibras e flavonoides, e são consumidos seus cladódios (popularmente entendidos como as folhas da planta), suas flores, sementes e frutos, de forma crua ou cozida (BEZERRA; BRITO, 2020). Os dados da pesquisa referentes as pessoas que consumiram palma, apesar de escassos, indicam que as pessoas são nascidas no Norte e Centro-oeste do país, regiões onde sua oferta é mais recorrente. Segundo Lucena (2016), boa parte da produção de palma é destinada a alimentação animal, principalmente de bovinos e ovinos, e a população, mesmo da região da caatinga, ainda tem preconceito com a inserção da palma na dieta humana. Apesar disso, a planta se mostra um bom substituto de alimentos convencionais, apresentando uma quantidade maior de fibras do que a laranja, e teores maiores de vitamina A, ferro e cálcio do que vegetais diariamente consumidos como o tomate, quiabo, vagem, pimentão, chuchu e a couve-flor.

Quando questionados em relação a distribuição das PANCs elencadas na presente pesquisa, $75,6 \%$ dos entrevistados responderam que têm acesso a pelo menos uma das plantas na região onde vivem, 4,8\% não têm acesso, e 19,7\% alegaram não saber. 58,8\% dos entrevistados alegaram que comprariam as PANCs listadas caso estivessem disponíveis para compra em mercados. $32,4 \%$ responderam que talvez comprariam e somente $8,8 \%$ não comprariam nenhuma das PANCs. A partir desses dados, podemos evidenciar que a falta de conhecimento e consumo de PANCs pode estar relacionada a sua baixa disponibilidade nos maiores centros de compra, sendo que esses tipos de plantas estão normalmente disponíveis em feiras livres, quitandas ou são provenientes de produtores locais. NarcisaOliveira et al. (2018) evidenciaram em seus estudos que as PANCs podem ser desconhecidas pela população em geral por falta de incentivo e políticas públicas de inserção das PANCs na alimentação, e a sociedade acaba ignorando o uso alimentar desses vegetais. Por consequência, esses vegetais acabam sendo marginalizados pela agroindústria.

Por fim, os entrevistados foram questionados sobre acreditarem nos benefícios da inclusão de PANCs na dieta, com relação a saúde e nutrição. $87,8 \%$ dos entrevistados 
acreditam que haveria benefícios, I0,8\% acreditam que possa haver benefícios em alguns casos e somente $1,4 \%$ não acreditam nos benefícios das PANCs. A pesquisa pode esclarecer que, para a maioria das pessoas, a inclusão das PANCs na dieta agregaria riqueza nutricional e funcional às refeições, uma vez que as pessoas tomem conhecimento sobre as PANCs e seus preparos para o consumo.

\section{${ }_{4}$ CONCLUSÃO}

O presente estudo permitiu evidenciar que o termo plantas alimentícias não convencionais ou PANCs não é do conhecimento da maioria da população pesquisada, porém grande parte teve contato com alguma PANC ou até já fez o consumo de algumas delas. Dentre as PANCs citadas na pesquisa, as mais consumidas foram hibisco, feijão guandu, ora-pro-nóbis e picão. As PANCs mais citadas podem ser encontradas na região onde os participantes residem, mas existe a falta de disponibilidade em grandes centros comerciais, como mercados. Pode-se perceber o interesse dos participantes na inclusão de PANCs na dieta e seus benefícios. O trabalho ainda deixa evidente que existe uma necessidade crescente de estudos relacionados a PANCs, de maneira que se crie uma base de dados sólida sobre a enorme diversidade de plantas e seus benefícios, para que, assim, a população possa decidir sobre a inclusão desses alimentos em suas dietas

\section{REFERÊNCIAS BIBLIOGRÁFICAS}

ABREU, N. C. O., CASTANHEIRA, J. D. As vantagens da introdução das plantas alimentícias não convencionais na alimentação dos beneficiários do bolsa família da estratégia saúde da família Bernardo Valadares, em Sete Lagoas-MG. Revista Brasileira de Ciências da Vida, v. 5, n. 4, 2017.

ALMEIDA, K., SCHWEIG, M. Elaboração de biscoitos sem glúten com farinha de hibisco e ricos em fibras. Trabalho de Conclusão de Curso (Tecnologia em Alimentos) Universidade Tecnológica Federal do Paraná, Medianeira, 2018. Disponível em: http://repositorio.roca.utfpr.edu.br/jspui/handle/r/I5592. 
BARBOSA, T. P. et al. Plantas alimentícias não convencionais: existem potenciais consumidores e locais para comprar? Research, Society and Development, v. Io, n. 4, 202I. Disponível em: https://rsdjournal.org/index.php/rsd/article/view/ı4I46.

BARREIRA, T. F. et al. Diversidade e equitabilidade de Plantas Alimentícias Não Convencionais na zona rural de Viçosa, Minas Gerais, Brasil. Revista Brasileira de Plantas Medicinais, Campinas, v.17, n.4, supl. II, p.964-974, 2015. Disponível em: https://www.scielo.br/j/rbpm/a/Y8H4bjxPnk${ }_{3}$ frsdGcZmRV 4 F/?lang=pt.

BEZERRA, J. A., BRITO, M. M. Potencial nutricional e antioxidante das plantas alimentícias não convencionais (PANCs) e o uso na alimentação: revisão. Research, Society and Development, v. 9, n. 9, 2020. Disponível em: https://rsdjournal.org/index.php/rsd/article/view/7159.

FERRARINI, M. E. H. et al. Popularizando as Plantas Alimentícias Não Convencionais em Botucatu-SP: Construção de saberes em busca da diversidade alimentar. Anais do XI Congresso Brasileiro de Agroecologia, v. 15, n. 2, 2020. Disponível em: http://cadernos.aba-agroecologia.org.br/index.php/cadernos/article/view/5766.

FONSECA, C. et al. A importância das plantas alimentícias não convencionais (PANCS) para a sustentabilidade dos sistemas de produção de base ecológica. Cadernos de Agroecologia. Anais do VI CLAA, X CBA e V SEMDF, v. I3, n. I, 2018. Disponível em: http://cadernos.aba-agroecologia.org.br/index.php/cadernos/article/view/r67. GONÇALVES, R. P., LIMA, J. R., MORAES, L. A. Hortas como formas de utilização e disseminação de plantas alimentícias não convencionais no Brasil. FTT Journal of 
Engineering and Business, São Bernardo do Campo, n. 6, p. 67-78, 2020. Disponível em: http://journal.ftt.com.br/seer/index.php/FTT/article/view/348.

JACOB, M. C. M. Biodiversidade de plantas alimentícias não convencionais em uma horta comunitária com fins educativos. DEMETRA: Alimentação, Nutrição \& Saúde, V. I5, p. I-I7, 2020. Disponível em: https://www.e-publicacoes.uerj.br/index.php/demetra/article/view/44037.

KINUPP, V. F. Plantas Alimentícias Não-Convencionais (PANCs): uma riqueza negligenciada. Anais da 6ı ${ }^{\mathrm{a}}$ Reunião Anual da SBPC, Manaus, 2009.

LUCENA, T. K. P. et al. Sabores de nossa terra: uma abordagem ecológica e nutricional de mandacaru (Cereus jamacaru) e de palma forageira (Opuntia ficus-indica) em âmbito escolar. Campina Grande, Editora Realize, 2016. Disponível em: https://www.editorarealize.com.br/artigo/visualizar/24163.

NARCISA-OLIVEIRA, J. et al. Plantas alimentícias não convencionais (PANCs) no município de Campo Grande/MS: Conhecimento popular, consumo e comércio. Cadernos de Agroecologia, v. 13, n. 2, 2018. Disponível em: http://cadernos.abaagroecologia.org.br/index.php/cadernos/article/view/r99o.

NEDOPETALSKI, P. F., KRUPEK, R. A. O uso de plantas medicinais pela população de União da Vitória - PR: o saber popular confrontado pelo conhecimento científico. Arquivos do Mudi, v. 24, n. I, p. 50-67, 2020. Disponível em: https://periodicos.uem.br/ojs/index.php/ArqMudi/article/view/51921. 
PRIETO, D. et al. Efecto de la administración de una infusión acuosa de Hibiscus

Sabdariffa L. sobre el índice de masa corporal, apetito y saciedad en individuos con obesidad grado I. Archivos Venezolanos de Farmacología y Terapéutica, Caracas, v. 32, n. 3,p. 34-40, 2013. Disponível em:

http://ve.scielo.org/scielo.php?script=sci_arttext\&pid=So798$02642013000300001 \& \operatorname{lng}=$ es $\&$ nrm $=$ iso.

REIS, J. P. G. et al. Estudo do emprego de plantas alimentícias não convencionais (PANCS): característica nutricional, propriedade funcional e emprego na alimentação humana. Anais I CONAPESC, Campina Grande, Realize Editora, 2or6. Disponível em: https://www.editorarealize.com.br/artigo/visualizar/ı0o9.

SILVA, P. R. J. et al. Plantas alimentícias não convencionais como alimento funcional: Uma revisão bibliográfica. Anais da Faculdade de Medicina de Olinda, v. I, n. 4, 2019. Disponível em: https://afmo.emnuvens.com.br/afmo/article/view/III. 